\title{
ENERGETICS OF PHOTOINDUCED CHARGE MIGRATION WITHIN THE TRYPTOPHAN TETRAD OF AN ANIMAL (6-4) PHOTOLYASE
}

Fabien Cailliez, ${ }^{a}$ Pavel Müller, ${ }^{b}$ Thiago Firmino, ${ }^{a}$ Pascal Pernot, ${ }^{a}$ Aurélien de la Lande ${ }^{a}$

[a] : Laboratoire de Chimie Physique, UMR 8000 CNRS/Univ. Paris-Sud, Univ. Paris Saclay, 91405 Orsay, France

[b] : Institute for Integrative Biology of the Cell (I2BC), CEA, CNRS, Univ. Paris-Sud, Univ. Paris-Saclay, 91198 Gif-sur-Yvette cedex, France

\section{SUPPLEMENTARY INFORMATION}

\section{List of supplementary Figures}

Figure S1: Root-Mean-Square-Deviation of XI(6-4)PL backbone atoms as a function of the simulation time. The MD trajectory was performed in the resting (ground) state.

Figure S2: Reorganization energies computed from the variance of energy gaps.

Figure S3: Effective driving force, reorganization energy and characteristic ET rate for the second ET step $\left(\operatorname{Trp}_{319} \rightarrow \operatorname{Trp}_{376^{\circ+}}\right)$

Figure S4: Effective driving force, reorganization energy and characteristic ET rate for the third ET step $\left(\operatorname{Trp}_{370} \rightarrow \operatorname{Trp}_{319}{ }^{\circ+}\right)$

Figure S5: Fluctuations of $\left|H_{D A}\right|$ for the first electron transfer step as a function of three geometrical parameters characterizing the relative orientation of $\operatorname{Trp}_{396}$ and $\operatorname{Trp}_{319}$.

Figure S6: Displacements of the normal modes of the tryptophan and the tryptophanyl radical cation upon oxidation and reduction respectively.

\section{Validation of the QM+MM approach}

\section{Uncertainty calculations}




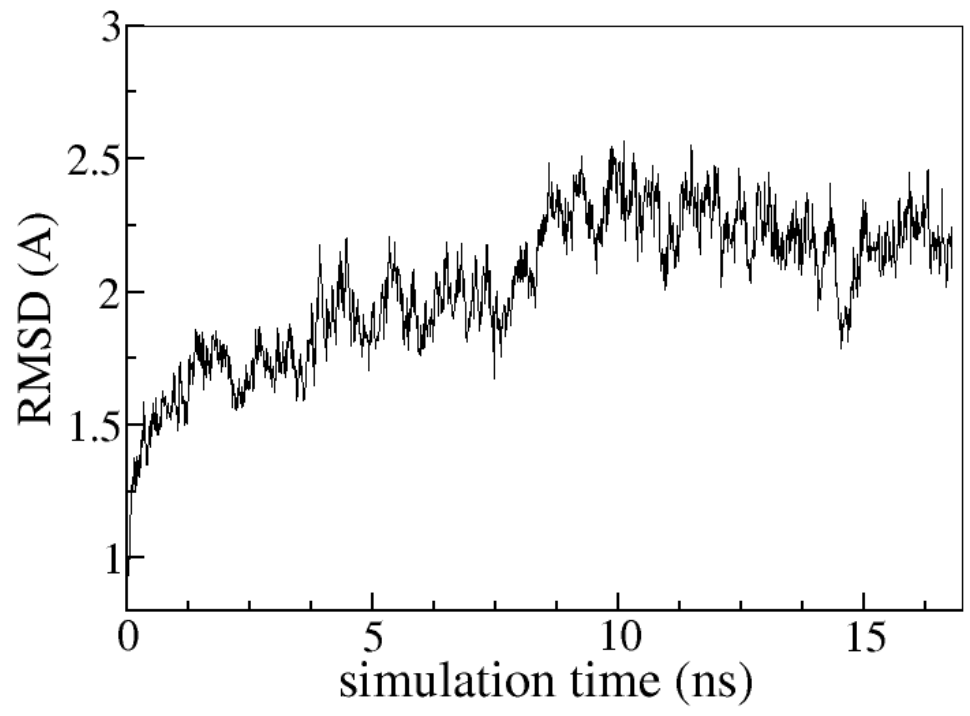

Figure S1: Root-Mean-Square-Deviation of $X /(6-4) P L$ backbone atoms as a function of the simulation time. The MD trajectory was performed in the resting (ground) state. 

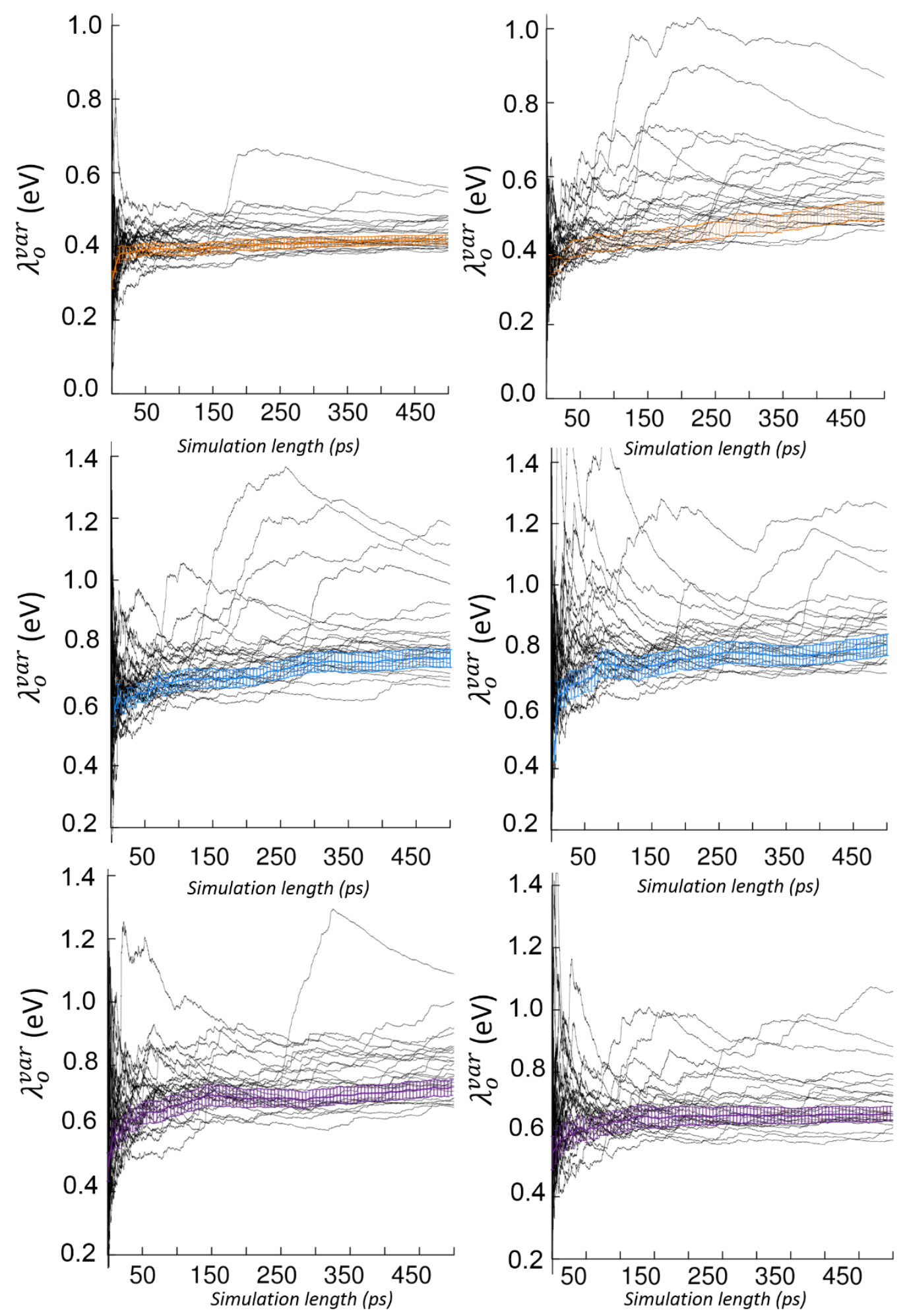

Figure S2: Reorganization energies computed from the variance of energy gaps. From top to bottom: $\mathrm{ET}^{1}, \mathrm{ET}^{2}$, and $\mathrm{ET}^{3}$. The left column correspond to calculations performed on the simulation performed on the initial state of the $\mathrm{ET}$, and right column on those performed on the final state of the ET. The colored curves correspond to average values over the 26 simulations. To avoid overwhelming information, statistical uncertainties are given only for the average curve. Details of the calculation of uncertainties are given below. 

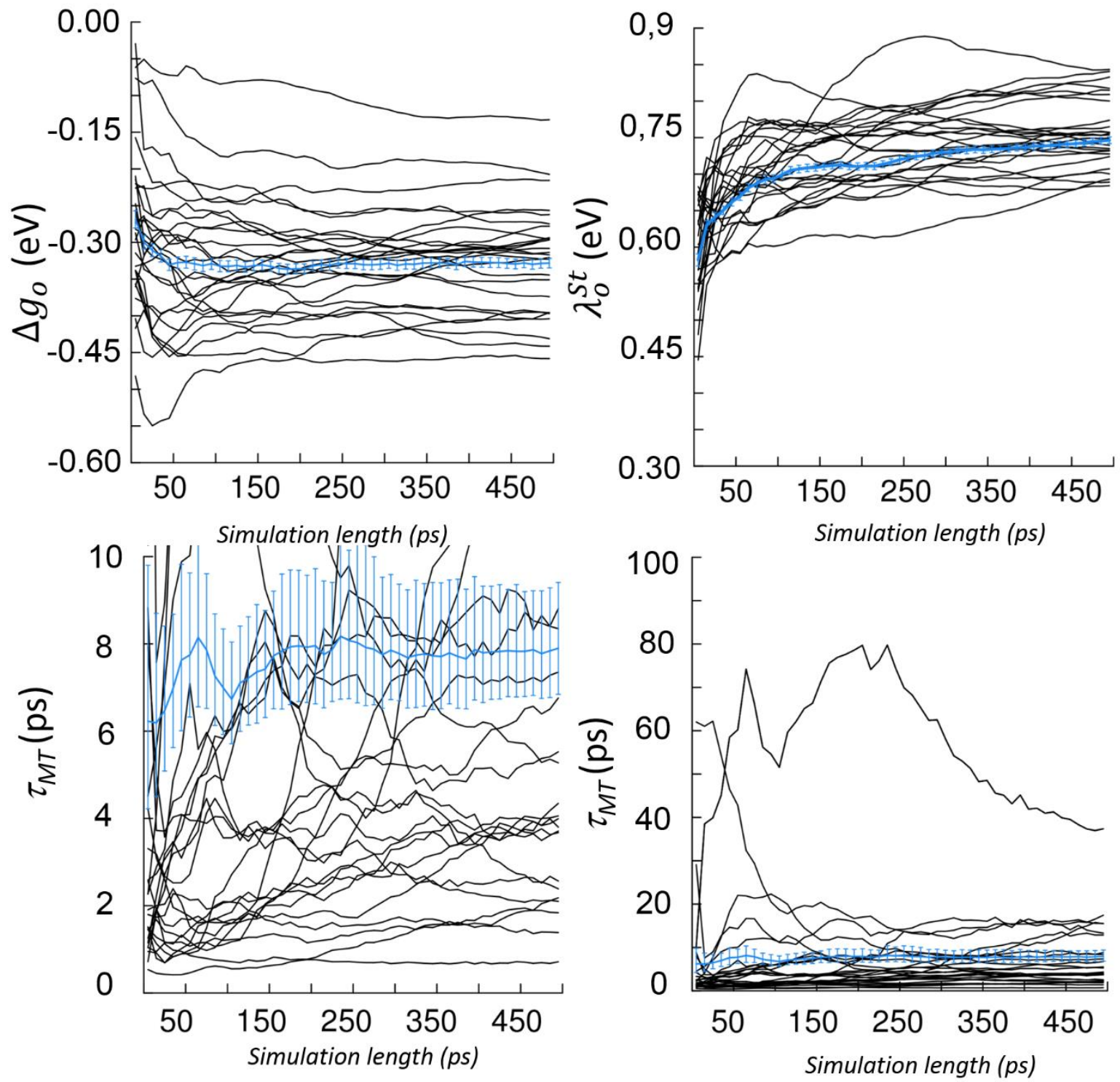

Figure S3: Top: restricted driving force and reorganization energy (in $\mathrm{eV}$ ) for the second electron transfer step from $\operatorname{Trp}_{319} \rightarrow \operatorname{Trp}_{376}{ }^{\circ+}$ calculated from 26 sets of MD trajectories. Bottom: characteristic ET time (in ps). Two plots with different scales are shown because of one set of MD trajectories leading a very large ET time. The blue curves are the average over the 26 individual contributions. To avoid overwhelming information, statistical uncertainties are given only for the average curve. Uncertainties of the individual curves are approximately $5(\simeq \sqrt{26})$ times larger. Details of the calculation of uncertainties are given below. 

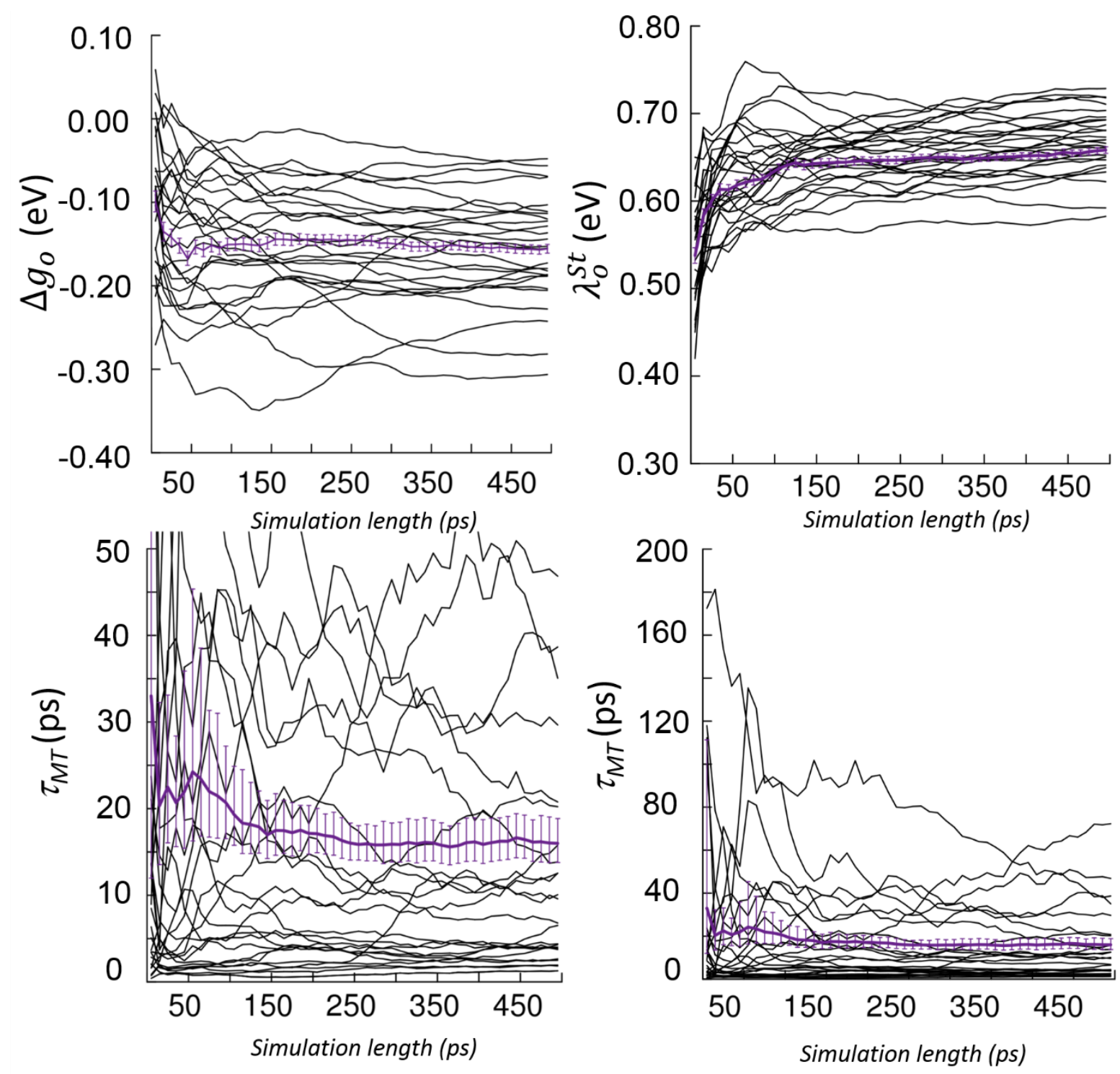

Figure S4: Top: restricted driving force and reorganization energy (in eV) for the third electron transfer step from $\operatorname{Trp}_{370} \rightarrow \operatorname{Trp}_{319}{ }^{\circ+}$ calculated from the 26 sets of MD trajectories. Bottom: characteristic ET time (in ps). Two plots with different scales are shown because of one set of MD trajectories leading a very large ET time. The violet curves are the average over the 26 individual contributions. To avoid overwhelming information, statistical uncertainties are given only for the average curve. Uncertainties of the individual curves are approximately $5(\simeq \sqrt{26})$ times larger. Details of the calculation of uncertainties are given below. 

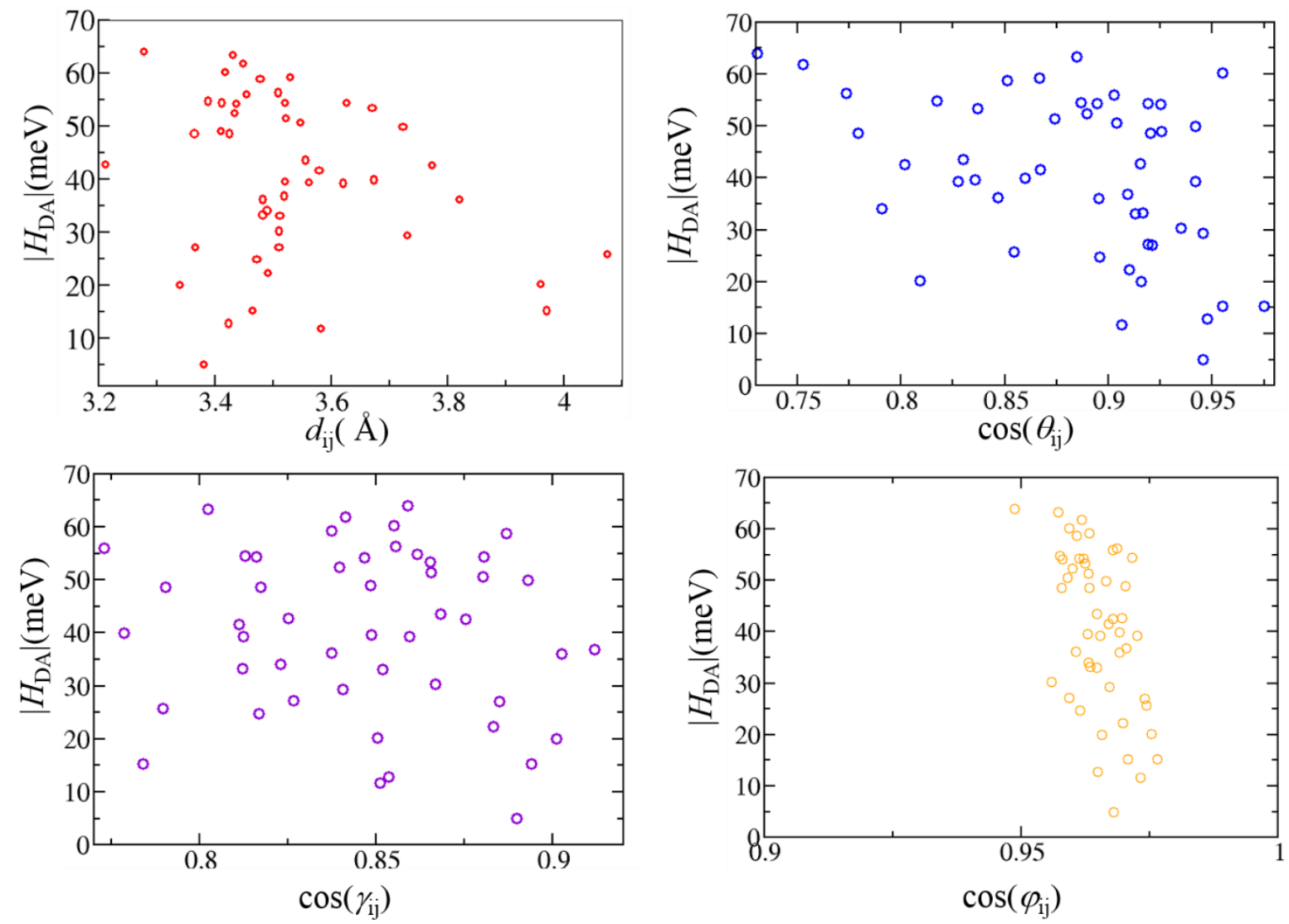

Figure S5: Fluctuations of $\left|H_{D A}\right|$ for the first electron transfer step as a function of three geometrical parameters defined in Scheme 2 (see main text). For clarity, we took only a subensemble of 50 configurations out of the 2600 available.



Figure S6: Displacements of the normal modes of the tryptophan and the tryptophanyl radical cation upon oxidation and reduction respectively. 


\section{Validation of the QM+MM approach}

As detailed in the main text, driving forces and reorganization energies are calculated using a $\mathrm{QM}+\mathrm{MM}$ scheme. This means that the inner- and outer-sphere contributions arising from the Trp cofactors (Trp) and from the environment (E), respectively, are calculated separately. The former are obtained from DFT calculations of a geometrically optimized methylindole molecule in vacuum in the neutral and cationic forms. The latter are calculated by sampling the Trp/E Coulomb and induction contributions to the potential vertical energy gap on the initial and final state with MD simulations.

Compared to a QM/MM scheme where hybrid DFT/MM calculations would be performed along classical MD simulations, the $\mathrm{QM}+\mathrm{MM}$ relies on two hypotheses. First that the polarization of the redox cofactors has negligible effects on the intrinsic propensity of the Trp and $\operatorname{Trp}^{\circ+}$ moieties to lose or accept an electron, respectively. Second, that structural dynamics of the Trp side chains in the protein are weakly dependent on the environment and would be similar in vacuum. We have verified the validity of these hypotheses taking the $\operatorname{Trp}_{377}$ $\rightarrow \operatorname{Trp}_{400^{\circ+}}$ electron transfer in Arabidopsis thaliana cryptochrome (AtCRY) as a test case. Results of this study will be reported elsewhere. ${ }^{1}$ We only give here values of the inner-sphere contribution to $\lambda_{i}^{S t}$ calculated from optimized geometries in vacuum or from constrained DFT/MM MD simulations of AtCRY. We also calculated inner-outer sphere cross term contribution $\lambda_{i-o}^{S t}$ to $\lambda_{i}^{\text {var }}{ }^{2}$ Note the hybrid cDFT/MM scheme employed an electrostatic embedding scheme, i.e., the Trp cofactors were polarized by the environment in the simulations. Results in Table S1 indicate that the influence of the polarization of the redox cofactors as well as the coupling between the redox active tryptophan side chains and the environment motions are of the order of $0.01 \mathrm{eV}$, therefore validating a QM+MM scheme for ET in this protein. One can reasonably assume that this conclusion can be generalized to other proteins from the CRYs and PLs superfamily, including XI(6-4)PL.

\begin{tabular}{|c|c|c|}
\hline$\lambda_{i-D F T / M M}^{S t}(\mathrm{eV})$ & $\lambda_{i-\text { vacuum }}^{S t}(\mathrm{eV})$ & $\lambda_{i-o-D F T / M M}^{\text {var }}(\mathrm{eV})$ \\
\hline 0.258 & 0.248 & 0.006 \\
\hline
\end{tabular}

Table S1: reorganization energy associated to the $\operatorname{Trp}_{377} \rightarrow \operatorname{Trp}_{400}{ }^{\circ+}$ electron transfer in AtCRY. Calculations performed with the PBE functional and DZVP-GGA basis set. Note that PBE produces smaller reorganization energies than B3LYP which is used in our study of $X /(6-4) \mathrm{PL}$. 


\section{Uncertainty calculations}

Uncertainty calculations are necessary to draw conclusions from computed data as well as from experimental ones. The methods used to estimate uncertainties on the various quantities discussed in the main text are described below. In what follows, $u(X)$ denotes the uncertainty of a quantity $X$, and $\operatorname{var}(X)$ represents the variance of it.

\section{Uncertainties of $\Delta g_{o}$ and $\lambda_{o}^{S t}$ from a single pair of MD trajectories.}

For a pair of MD trajectories in electronic states 1 and 2, the uncertainties of $\Delta g_{r s t r}$ and $\lambda_{r s t r}^{S t}$ are computed using propagation uncertainty formula, from the variance of the mean vertical energy gaps $\langle\Delta E\rangle_{i}$ along the two trajectories:

$$
u^{2}\left(\Delta g_{o}\right)=u^{2}\left(\lambda_{o}\right)=\frac{1}{4}\left(u^{2}\left(\langle\Delta E\rangle_{1}\right)+u^{2}\left(\langle\Delta E\rangle_{2}\right)\right)
$$

The variance of the mean energy gaps are obtained from the dispersion of the energy gaps along the simulations:

$$
u^{2}\left(\langle\Delta E\rangle_{i}\right)=\frac{\operatorname{var}(\Delta E)_{i}}{\rho_{i} N}
$$

$N$ is the number of points used for evaluating the mean energy gap and $\rho_{i}$ is the statistical chain efficiency of the MD simulation $i$. The latter quantity has been obtained from the energy gap auto-correlation function using the effectiveSize procedure of the coda package of the $R$ software. This leads finally to:

$$
u^{2}\left(\Delta g_{o}\right)=u^{2}\left(\lambda_{o}\right)=\frac{1}{4}\left(\frac{\operatorname{var}(\Delta E)_{1}}{\rho_{1} N}+\frac{\operatorname{var}(\Delta E)_{2}}{\rho_{2} N}\right)
$$

Uncertainties on the reorganization energies $\lambda^{\text {var }}$ computed from the variance of the energy gaps

For each simulation (on electronic state 1 or 2), one can compute the reorganization energy from the variance of the energy gap $\Delta E=E_{2}-E_{1}$, according Eq. 5 of the main text:

$$
\lambda_{i}^{\text {var }}=\frac{\operatorname{var}(\Delta E)_{i}}{2 k_{B} T}
$$

Where the index $i$ represents the electronic state on which the value is computed. As stated in the main text, if the Linear Response Approximation applies, the three ways to compute the reorganization energy lead to the same value $\lambda^{S t}=\lambda_{1}^{\text {var }}=\lambda_{2}^{v a r}$.

Uncertainty on each individual $\lambda_{i}^{\text {var }}$ value is estimated through: ${ }^{3}$

$$
u^{2}\left(\lambda_{i}^{v a r}\right)=\left(\frac{1}{2 k_{B} T}\right)^{2} \frac{2\left(\operatorname{var}(\Delta E)_{i}\right)^{2}}{\rho_{i} N}
$$

Uncertainties of the average $\overline{\Delta g_{o}}, \overline{\lambda_{o}^{S t}}$, and $\overline{\lambda_{l}^{v a r}}$ from the ensemble of MD trajectories.

In order to take into account the variability of the uncertainty of $\Delta g_{o}$ and $\lambda_{o}^{S t}$ in the 26-series of $\mathrm{MD}$ simulations, we have computed weighted average values for these quantities, according to: 
where $M$ is a normalization factor

$$
\overline{\Delta g_{o}}=\frac{1}{M} \sum_{k=1}^{26} \frac{1}{u^{2}\left(\Delta g_{o}^{k}\right)} \Delta g_{o}^{k}
$$

$$
M=\sum_{k=1}^{26} \frac{1}{u^{2}\left(\Delta g_{o}^{k}\right)}
$$

This procedure assigns more weight to sets of MD trajectories with smaller uncertainties.

The uncertainty associated with $\overline{\Delta g_{o}}$ is calculated by propagating the uncertainties on the individual $\Delta g_{o}^{k}$ values, under the assumption that they are uncorrelated (because they are computed from independent MD simulations). This leads to:

$$
u^{2}\left(\overline{\Delta g_{o}}\right)=\frac{1}{M}
$$

The two expressions used to calculate the mean value and uncertainty for the effective driving force $g_{r s t r}$ are also used to compute mean value and uncertainty for the $\lambda_{o}^{S t}$ or $\lambda_{i}^{v a r}$.

\section{Uncertainties on the electronic coupling}

As stated in the main text, mean values of the unsigned electronic coupling $\left\langle\left|H_{D A}\right|\right\rangle_{i}$ have been computed for each electronic state $i$ as the average value over the data from the $26 \mathrm{MD}$ simulations performed over that electronic state:

$$
\left\langle\left|H_{D A}\right|\right\rangle_{i}=\frac{1}{26} \sum_{k=1}^{26}\left\langle\left|H_{D A}(k)\right|\right\rangle_{i}=\frac{1}{26 \times 50} \sum_{k=1}^{26} \sum_{j=1}^{50}\left|H_{D A, i}(j, k)\right|
$$

Where $H_{D A, i}(j, k)$ is the electronic coupling for one snapshot $j$ extracted from the $k^{\text {th }}$ simulation performed on electronic state $i$.

The uncertainty associated with this value has been computed as a block average over the 26 trajectories:

$$
u^{2}\left(\left\langle\left|H_{D A}\right|\right\rangle_{i}\right)=\frac{1}{26} \sum_{k=1}^{26}\left(\left\langle\left|H_{D A}(k)\right|\right\rangle_{i}-\left\langle\left|H_{D A}\right|\right\rangle_{i}\right)^{2}
$$

\section{Uncertainties on the electron transfer characteristic times $\tau_{M T}$}

For each ET, the characteristic time is defined as the average over the 26 characteristic times computed from Marcus expression (Eq. 2 of the main text), rewritten as:

$$
=\frac{1}{26} \sum_{i=1}^{26} \sum_{v}\left[\frac{2 \pi\left\langle\chi_{0} \mid \chi_{v}\right\rangle^{2}}{\hbar \sqrt{2 \pi k_{B} T}} \frac{H_{D A}^{2}(i)}{\sqrt{\left(\langle\Delta E(i)\rangle_{1}-\langle\Delta E(i)\rangle_{2}\right)}} \exp \left(-\frac{\left(\langle\Delta E(i)\rangle_{1}+\Delta \mathrm{E}_{v i b}\right)^{2}}{2\left(\langle\Delta E(i)\rangle_{1}-\langle\Delta E(i)\rangle_{2}\right) k_{B} T}\right)\right]^{-1} \text { (Eq }
$$

Where $H_{D A}^{2}(i),\langle\Delta E(i)\rangle_{1}$, and $\langle\Delta E(i)\rangle_{2}$ are the electronic coupling, and the mean energy gaps on electronic state 1 or 2 respectively, calculated from MD simulations performed from the starting point $i$. Like in Eq. 2 the loop over the index $v$ refers to the inner-sphere contributions. Each term of the sum differs by the value of the Franck-Condon factor $\left(\left\langle\chi_{0} \mid \chi_{v}\right\rangle^{2}\right)$ and the vibrational energy $\left(\Delta \mathrm{E}_{v i b}\right)$. 
Due to the non-Gaussian shape of electronic coupling distribution, we have computed ET characteristic times mean values and uncertainties using a Monte Carlo approach instead of using uncertainty propagation formula. To this end, we computed an average of 5000 values with (Eq. S1) and reported the average value and the $99 \%$ confidence interval in Figures 2 , S3, and S4. For each evaluation, the electronic coupling is taken from the distribution of $H_{D A}$ values obtained during the simulation, whereas the average energy gaps are randomly chosen from normal distributions the mean and standard deviations of which are estimated from MD simulations data.

\footnotetext{
${ }^{1}$ Firmino, Th.; Mangaud, E.; Cailliez, F.; Desouter-Lecomte, M.; de la Lande, A. in preparation

2 Ando, K. J. Chem. Phys. 2001, 114, 9470.

${ }^{3}$ Statistical Inference; 2nd ed.; Casella, G.; Berger, R., L., Eds. Pacific Grove CA, 2001.
} 